\title{
Efeito da talidomida em paciente com hemorragia secundária a ectasia vascular de antro gástrico dependente crônico de transfusões
}

\section{Chronic transfusion-dependent patient with gastric antral vascular ectasia successfully treated with thalidomide}

Samara Jacinto de Limaํ․ Lúcia Libanês Bessa Campelo Braga². Marcellus Henrique Loiola Ponte ${ }^{3}$. José Milton de Castro Lima ${ }^{4}$. Matthaus Rabelo da Costa ${ }^{5}$.

1 Médica, Universidade Federal do Ceará (UFC), Residência em Clínica Médica pelo Hospital Geral de Fortaleza (HGF), Fortaleza, Ceará, Brasil. 2 Professora Titular de Gastroenterologia Departamento de Medicina Clínica da Faculdade de Medicina-UFC, Doutora em Gastroenterologia, Chefe do Serviço de Gastroenterologia Hospital Universitário Walter Cantídio (HUWC)-UFC, Supervisora da Residência de Gastroenterologia-HUWC, Fortaleza, Ceará, Brasil. 3 Professor Associado do Departamento de Medicina Clínica da Faculdade de Medicina-UFC, Gastroenterologista, Especialista em Endoscopia Digestiva e Doutor em Clínica Médica. Supervisor da residência em Endoscopia Digestiva do Hospital Universitário Wálter Cantídio-UFC, Fortaleza, Ceará, Brasil. 4 Professor Associado do Departamento de Medicina Clínica da Faculdade de Medicina da Universidade Federal do Ceará (UFC), Doutor em Gastroenterologia. Coordenador do Ambulatório de Hepatites Agudas e Crônicas do Hospital Universitário Walter Cantídio-UFC, Fortaleza, Ceará, Brasil. 5 Acadêmico de medicina, Universidade Federal do Ceará.

\section{RESUMO}

A talidomida é uma droga com efeito anti-inflamatório, imunomodulador e antiangiogênico. Devido a isso, vêm sendo publicados diversos relatos de casos, descrevendo sua eficácia no tratamento de hemorragia digestiva secundária à angiodisplasias do trato gastrointestinal, à doença inflamatória intestinal e um caso de resposta clínica na hemorragia secundária à ectasia vascular de antro gástrico (GAVE). No presente trabalho, relatamos o caso de uma paciente de 52 anos, com cirrose hepática e ectasia vascular do antro gástrico, que apresentou seis episódios de hemorragia digestiva alta com necessidade de transfusão sanguínea, apesar da terapia endoscópica estabelecida. Iniciamos talidomida na dose de $100 \mathrm{mg} /$ dia que em seguida foi reduzida para $50 \mathrm{mg} / \mathrm{dia}$. A paciente evoluiu com melhora clínica significativa, não apresentando novos episódios de sangramento, nem necessidade de transfusões sanguíneas. Apesar da interrupção da terapêutica endoscópica, persistiu assintomática e a endoscopia de controle após 3 meses não visualizava mais sangramento ativo nas lesões do antro. Portanto, este trabalho tem como objetivo descrever que a talidomida se mostrou eficaz, em baixa dose, para o controle do sangramento secundário à GAVE nesta paciente.

Palavras-chave: Talidomida. Sangramento. Ectasia vascular do antro gástrico. Coagulação com plasma de argônio.

\section{ABSTRACT}

Thalidomide is a drug with anti-inflammatory, immunomodulatory, and anti-angiogenic effect. Several case reports have been published describing its effectiveness in the treatment of digestive hemorrhage secondary to angiodysplasias, inflammatory bowel disease, and one case of clinical response in hemorrhage secondary to the gastric antral vascular ectasia (GAVE). The present study report the case of a patient with hepatic cirrhosis and presence of GAVE, who underwent six episodes of upper gastrointestinal bleeding requiring blood transfusion, despite the established endoscopic therapy. We started thalidomide at a dose of $100 \mathrm{mg} /$ day, which was decreased to $50 \mathrm{mg}$ /day. The patient evolved with significant clinical improvement, without any new episode of bleeding or need for blood transfusions. In spite of discontinuation of the endoscopic therapy, she persisted asymptomatic and endoscopy control did not visualize active bleeding. Therefore, thalidomide has shown effective, at low dose, for control of bleeding secondary to GAVE in this patient.

Keywords: Thalidomide. Bleeding. Gastric antral vascular ectasia. Argon plasma coagulation.

Autor correspondente: Samara Jacinto de Lima, Avenida Soriano Albuquerque, 831, Joaquim Távora, Fortaleza, Ceará. CEP: 60130-160.Telefone: +5585 99710-6767. E-mail: samarajacinto2004@hotmail.com

Conflito de interesses: Não há qualquer conflito de interesses por parte de qualquer um dos autores.

Recebido em: 18 Fev 2015; Revisado em: 04 Mar 2016; Aceito em: 22 Mar 2016. 


\section{INTRODUÇÃO}

A ectasia vascular do antro gástrico (GAVE) é uma causa incomum de hemorragia gastrointestinal não varicosa. ${ }^{1}$ Pacientes com GAVE podem manifestar sintomas e sinais de hemorragia digestiva alta ou perdas ocultas de sangue, apresentando anemia e deficiência de ferro. ${ }^{1}$ A GAVE é facilmente diagnosticada durante exame endoscópico.

A fisiopatologia da GAVE não é totalmente esclarecida, no entanto a hipertensão portal não parece ser fundamental para o desenvolvimento da GAVE. ${ }^{2}$ A insuficiência hepática parece ter papel mais significativo, uma vez que esta condição é observada mais frequentemente em pacientes com doença hepática mais grave e tem mostrado ser resolvida após o transplante hepático.

A base da terapia na GAVE é a ablação endoscópica das lesões. Em pacientes refratários às técnicas endoscópicas, a talidomida, pelo seu efeito antiangiogênico, foi descrita anteriormente como terapia alternativa no tratamento da GAVE. A seguir, relataremos o caso de uma paciente com anemia ferropriva refratária com necessidade de múltiplas transfusões, secundária à hemorragia associada à GAVE, que apresentou melhora clínica com o uso da talidomida em baixas doses.

\section{RELATO DE CASO}

Descrevemos o caso de uma paciente feminina, 52 anos, proveniente de Manaus-AM, acompanhada desde 2008, com diagnóstico de cirrose hepática secundária à hepatite autoimune. Relatava internamento prévio em dezembro de 2012, por episódio de hemorragia digestiva alta (HDA) secundária à varizes esofágicas com necessidade de transfusão, tendo sido submetida a ligadura elástica. Apresentou dois novos episódios de hemorragia digestiva alta no início de 2013, com instabilidade hemodinâmica secundários a ectasias vascular de antro gástrico diagnosticadas durante exame endoscópico.

A paciente foi admitida no nosso serviço em abril de 2013, apresentando novo episódio de HDA. Realizou endoscopia digestiva alta (EDA) com visualização de micro-ectasias vasculares difusas de cárdia, corpo distal e antro gástrico com sinais de sangramento, além de varizes esofágicas erradicadas. Acompanhada com tratamento endoscópico de maio a julho de 2013, totalizando 4 sessões, inicialmente com plasma de argônio que, em seguida, foi associado à terapia com ligadura com bandas elásticas. Apesar da terapia endoscópica, apresentou seis episódios de melena, hematêmese e anemia, com necessidade de transfusões sanguíneas.

Em 26 de junho de 2013, a paciente foi admitida pela última vez com hemoglobina de 7,54 g/dL e apresentando melena. Nova EDA revelou ectasias vasculares gástricas com focos de sangramento difuso. A ligadura elástica foi realizada como terapia paliativa, mas não se obteve bons resultados. A EDA de controle, em julho de 2013, observava no esôfago sequelas de ligaduras elásticas prévias, enquanto no estômago haviam ectasias vasculares com pontos de sangramento ativo. Devido à anemia refratária, com níveis de hemoglobina variando de $6,8 \mathrm{~g} / \mathrm{dL}$ a $8,7 \mathrm{~g} / \mathrm{dL}$, houve a necessidade de tranfusões, totalizando 8 concentrados de hemáceas. Os episódios de hemorragia foram significativos, levando em dois deles à instabilidade hemodinâmica.

Pela refratariedade ao tratamento endoscópico e pelos relatos prévios do uso da talidomida em pacientes com hemorragia em trato gastrointestinal, optamos por associar a talidomida na dose de $100 \mathrm{mg} /$ dia logo após alta hospitalar, em 5 de agosto de 2013, concomitante à assinatura do termo de consentimento livre e esclarecido. A paciente foi reavaliada ambulatorialmente após duas semanas, sem queixas de melena ou hematêmese e com nível de hemoglobina de 10,2 g/dL. Ajustamos a dose da talidomida para $50 \mathrm{mg} / \mathrm{dia}$, mesmo na ausência de eventos adversos, por se tratar de uma paciente com cirrose hepática e embora seja extremamente raro, há relato de hepatotoxicidade como evento adverso à esta medicação. Investigou-se também, no acompanhamento ambulatorial, sintomas ou alterações ao exame físico, que pudessem sugerir neuropatia, entretanto a paciente também não apresentou esse efeito colateral.

Após início da talidomida, em novembro de 2013, nova EDA mostrou sinéquias pós-erradicação de varizes esofágicas e ectasias vasculares de antro gástrico sem sinais de sangramento. Prosseguimos o acompanhamento ambulatorial até janeiro de 2014, mantendo a terapia apenas com o uso da talidomida na dose de $50 \mathrm{mg} /$ dia, sem relatos de novos episódios de HDA e com estabilização dos níveis de hemoglobina (Gráfico 1).

A paciente foi transplantada em 15 de janeiro de 2014, após Modelo de Doença Hepática Terminal (MELD) corrigido por diagnóstico de hepatocarcinoma. Após transplante hepático, foi suspenso o uso da talidomida. A paciente segue em consultas ambulatoriais sem novos episódios de sangramento e com estabilização dos níveis de hemoglobina.

\section{DISCUSSÃO}

Inicialmente, acreditava-se que a GAVE era uma patologia rara, mas recentemente descobriu-se que ela é responsável por $4 \%$ dos casos de hemorragias digestivas altas não-varicosas, diagnosticadas em EDA. ${ }^{3}$ É descrita em $2 \%$ dos pacientes que aguardam transplante hepático. ${ }^{2}$ A maioria dos casos é idiopática, porém é frequentemente associada a outras condições clínicas, incluindo cirrose, esclerose sistêmica, diabetes mellitus, hipotireoidismo, insuficiência renal crônica e doença cardiovascular. ${ }^{4}$

A base terapêutica da GAVE é a ablação endoscópica das lesões. Existem diferentes abordagens endoscópicas terapêuticas no contexto da GAVE, no entanto a maioria dos estudos tem avaliado a utilização das técnicas termoablativas. ${ }^{4}$ Normalmente, várias sessões são necessárias para alcançar o controle do sangramento agudo e para corrigir a anemia devido à perda crônica de sangue.

Uma das terapias endoscópicas é o laser de coagulação $\mathrm{Nd}$ : 
Gráfico 1. Número de concentrados de hemácias transfundidos ao longo do tempo, correlacionando com o início do tratamento com a talidomida.

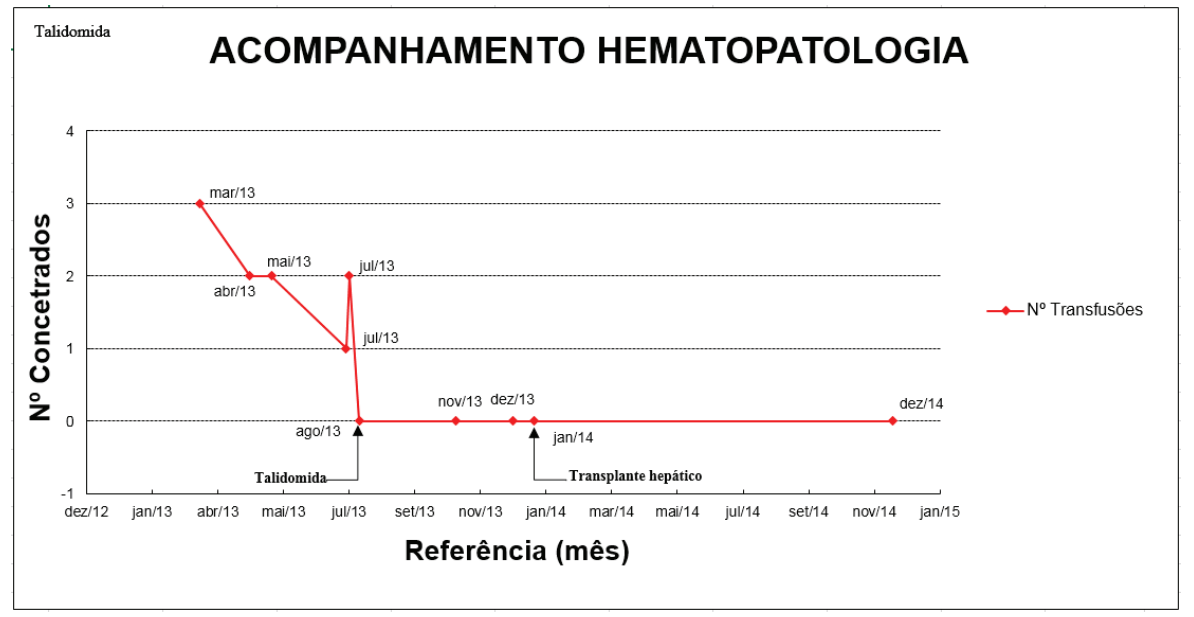

Fonte: elaborado pelos autores.

YAG (neodímio-ítrio-alumínio), que tem se mostrado eficaz em reduzir e inclusive abolir, em alguns casos, a necessidade de transfusões sanguíneas, após uma média de três sessões terapêuticas. ${ }^{5}$ A complicação mais grave é a perfuração gástrica, podendo haver ainda retardo do esvaziamento gástrico, estenose do piloro e formação de pólipos hiperplásicos. ${ }^{6}$

Atualmente, a terapia endoscópica de escolha para GAVE é a coagulação com plasma de argônio (APC). ${ }^{7}$ Um estudo relevante com APC relatou eficácia de $90 \%$ a $100 \%$, evitando a necessidade de transfusões sanguíneas e aumentando os níveis de hemoglobina em cerca de $3 \mathrm{~g} / \mathrm{dL} .^{7}$ Apesar dos bons resultados descritos nesse estudo, pode haver recorrência de sangramento em $30-60 \%$ dos casos, a médio e longo prazo. ${ }^{7}$

Wells et al. também relataram uma série de casos incluindo nove pacientes, que comparou a ligadura com bandas elásticas (EBL) com as terapias termoablativas (sonda térmica bipolar e o APC). Foram avaliados o risco de ressangramento e a necessidade de transfusão após o procedimento. ${ }^{8} \mathrm{~A}$ EBL mostrou-se superior nesse estudo. ${ }^{8}$ Apesar desses resultados, é necessário estudos com um número maior de pacientes para corroborar esses dados.

Quando a terapia endoscópica não for eficaz ou não for acessível, pode-se recorrer a outras opções para as quais existe uma experiência ainda muito limitada. Alguns relatos de casos descrevem que pacientes com GAVE obtiveram resposta clínica com o uso de octreotide, ${ }^{9}$ ácido tranexâmico ${ }^{10}$ e talidomida. ${ }^{11}$

A talidomida é uma opção terapêutica recente e promissora no tratamento das hemorragias digestivas, incluindo doentes com anemia secundária a malformações vasculares. Isto decorre do seu potente efeito antiangiogênico, que inibe o fator de crescimento vascular endotelial (VEGF) e reduz significativamente os seus níveis. ${ }^{12}$ Foi demonstrado que a inibição do VEGF depende da dose utilizada e de acordo com a literatura a dose da talidomida varia de 100-300 mg/dia, devendo-se manter seu uso por um período de 1 a 6 meses. ${ }^{12}$

A paciente descrita neste relato de caso apresentava cirrose hepática e GAVE, evoluindo com múltiplos episódios de HDA, que resultaram em anemia ferropriva e necessidade de múltiplas transfusões. Apesar da terapia endoscópica estabelecida, a paciente permaneceu refratária. Optamos por iniciar talidomida em doses de $100 \mathrm{mg} /$ dia, que, em seguida, foi reduzida para $50 \mathrm{mg} / \mathrm{dia}$. A paciente apresentou melhora clínica e laboratorial, sem necessidade de novas transfusões ou sessões de terapia endoscópica durante o acompanhamento de 6 meses. Após esse período, foi submetida a um transplante hepático e permaneceu assintomática até o momento.

Uma das limitações do uso da talidomida são seus efeitos colaterais. Além de seu significativo potencial teratogênico, são descritos sedação, constipação, erupções cutâneas e neuropatia periférica. A hepatotoxicidade é descrita como evento adverso extremamente raro, embora um número limitado de estudos tenham demonstrado que pode resultar em um evento adverso potencialmente grave. ${ }^{13}$ Após ser reintroduzida no mercado em 1988, indentificou-se um caso de morte diretamente associado ao tratamento com talidomida. ${ }^{14}$ Também foi descrito na Mayo Clinic, um caso de insuficiência hepática fulminante induzida por talidomida, que resultou na morte de uma paciente de 64 anos. ${ }^{15}$ Ressaltamos que apesar da hepatopatia de base da paciente descrita em nosso relato, não houve qualquer piora da função hepática após a introdução da talidomida.

Portanto, o tratamento da GAVE é ainda um desafio, uma vez que alguns doentes permanecem com episódios de HDA a despeito da terapia endoscópica. A relevância do caso descrito se dá em destacar a possibilidade do uso da talidomida em indivíduos com hemorragia digestiva alta secundária à GAVE e refratários às terapias já estabelecidas. Neste relato de caso, a paciente apresentou melhora clínica e laboratorial, que possivelmente pode estar associada ao efeito da talidomida. No entanto, consideramos que a indicação da talidomida em portadores de GAVE é controversa, fazendo-se necessários novos estudos com um maior número de pessoas, a fim de avaliar o verdadeiro mecanismo de ação da talidomida no tratamento da GAVE, bem como definir os riscos e benefícios da sua associação na terapia desses pacientes. 


\section{REFERÊNCIAS}

1. Selinger CP, Ang YS. Gastric antral vascular ectasia (GAVE): an update on clinical presentation, pathophysiology and treatment. Digestion. 2008;77(2):131-7.

2. Ward EM, Raimondo M, Rosser BG, Wallace MB, Dickson RD. Prevalence and natural history of gastric antral vascular ectasia in patients undergoing orthotopic liver transplantation. J Clin Gastroenterol. 2004;38(10):898-900.

3. Vincent C, Pomier-Layrargues G, Dagenais M, Lapointe R, Létourneau R, Roy A, et al. Cure of gastric antral vascular ectasia by liver transplantation despite persistent portal hypertension: a clue for pathogenesis. Liver Transpl. 2002;8(8):717-20.

4. Chaves DM, Sakai P, Oliveira CV, Cheng S, Ishioka S. Watermelon stomach: clinical aspects and treatment with argon plasma coagulation. Arq. Gastroenterol. 2006;43(3): 191-5.

5. Gostout CJ, Ahlquist DA, Radford CM, Viggiano TR, Bowyer BA, Balm RK. Endoscopic laser therapy for watermelon stomach. Gastroenterology 1989;96:1462-1465.

6. Mathou NG, Lovat LB, Thorpe SM, Bown SG. Nd: YAG laser induces long-term remission in transfusion-dependent patients with watermelon stomach. Lasers Med Sci. 2004;18(4):213-8.

7. Yusoff I, Brennan F, Ormonde D, Laurence B. Argon plasma coagulation for treatment of watermelon stomach. Endoscopy. 2002;34(5):407-10.

8. Kumar R, Mohindra S, Pruthi HS. Endoscopic band ligation: a novel therapy for bleeding gastric antral vascular ectasia. Endoscopy. 2007;39 Suppl 1:E56-E57.

9. Nardone G, Rocco A, Balzano T, Budillon G. The efficacy of octreotide therapy in chronic bleeding due to vascular abnormalities of the gastrointestinal tract. Aliment Pharmacol Ther. 1999;13(11):1429-36.

10. McCormick PA, Ooi H, Crosbie O. Tranexamic acid for severe bleeding gastric antral vascular ectasia in cirrhosis. Gut. $1998 ; 42(5): 750-2$.

11. Dunne KA, Hill J, Dillon JF. Treatment of chronic transfusiondependent gastric antral vascular ectasia (watermelon stomach) with thalidomide. Eur J Gastroenterol Hepatol. 2006;18(4):455-6.

12. Ge ZZ, Chen HM, Gao YJ, Liu WZ, Xu CH, Tan HH, et al. Efficacy of thalidomide for refractory gastrointestinal bleeding from vascular malformations. Gastroenterology. 2011;141(5):1629-37.

13. Hanje A, Shamp JL, Thomas FB, Meis GM. Thalidomide-induced severe hepatotoxicity. Pharmacotherapy. 2006;26(7):1018-22.

14. Clark TE, Edom N, Larson J, Lindsey LJ. Thalomid $\mathbb{R}$ (Thalidomide) Capsules: a review of the first 18 months of spontaneous postmarketing adverse event surveillance, including off-label prescribing. Drug Saf. 2001;24(2):87-117.

15. Hamadani M, Benson DM Jr, Copelan EA. Thalidomide-induced fulminant hepatic failure. Mayo Clin Proc. 2007;82(5):638-9.

\section{Como citar:}

Lima SJ, Braga LL, Ponte MH, Lima JM, Costa MR. Efeito da talidomida em paciente com hemorragia secundária à ectasia vascular de antro gástrico dependente crônico de transfusões. Rev Med UFC. 2016 jan-jun;56(1):64-67. 